\title{
Correlation between chemotherapy resistance in osteosarcoma patients and PAK5 and Ezrin gene expression
}

\author{
QIAN LIU*, BO XU* and WANSHAN ZHOU \\ Department of Surgery, Dezhou People's Hospital, Dezhou, Shandong 253014, P.R. China
}

Received July 19, 2017; Accepted October 24, 2017

DOI 10.3892/ol.2017.7355

\begin{abstract}
The correlation between PAK5 (P21-activated kinase 5) and Ezrin gene expression and chemotherapy resistance of osteosarcoma patients was investigated. The cisplatin (CDDP)-resistance model of osteosarcoma cells SOSP-9607/CDDP was established to detect the cell growth curve. Methyl thiazolyl tetrazolium (MTT) assay was used to detect the drug resistance of cells to chemotherapy drugs. Transwell assay was used to detect the invasive capacity of cells. Semi-quantitative PCR (qPCR) was used to detect the mRNA expression levels in the drug resistance-related genes PAK5 and Ezrin. Western blot analysis was used to detect the protein expression levels in PAK5 and Ezrin. Tumor tissues were taken from the osteosarcoma patients with chemotherapy resistance to detect the expression levels of PAK5 and Ezrin via immunohistochemical detection, and the correlation between PAK5 and Ezrin expressions was studied. The results of MTT assay showed that the growth rate of SOSP-9607 was similar to that of SOSP-9607/CDDP, and the difference was not statistically significant $(\mathrm{P}>0.05)$. The sensitivity of SOSP-9607 to CDDP was significantly higher than that of SOSP-9607/CDDP, and the difference was statistically significant $(\mathrm{P}<0.01)$. Transwell assay showed that the migration capacity of SOSP-9607/CDDP was significantly better than that of SOSP-9607 $(\mathrm{P}<0.01)$, indicating that the drug resistance cell lines of osteosarcoma were constructed successfully. Semi-qPCR and western blot analysis showed that the protein expression levels in PAK5 and Ezrin in SOSP-9607/CDDP were significantly higher than those in SOSP-9607 $(\mathrm{P}<0.01)$. The results of immunohistochemistry showed that the expression quantities of PAK5 and Ezrin in osteosarcoma tissues were significantly higher than those in
\end{abstract}

Correspondence to: Dr Wanshan Zhou, Department of Surgery, Dezhou People's Hospital, 1751 Xinhu Avenue, Dezhou, Shandong 253014, P.R. China

E-mail: wangshang5569@163.com

*Contributed equally

Key words: osteosarcoma, P21-activated kinase 5, Ezrin, drug resistance para-tumor tissues $(\mathrm{P}<0.01)$. Pearson's correlation analysis showed that expression of PAK5 and Ezrin was positively correlated $(\mathrm{r}=0.197, \mathrm{P}=0.023)$. The osteosarcoma resistance is closely related to the expression levels of PAK5 and Ezrin genes. Thus, PAK5 and Ezrin genes may affect the tolerance of osteosarcoma patients to chemotherapy drugs during treatment via the synergistic effect.

\section{Introduction}

Osteosarcoma, as a kind of malignant tumor derived from malignant interstitial cells, has certain osteoid characteristics, such as strong migration capacity and frequent systemic metastasis (1). The pathogenesis of osteosarcoma remains unclear and the osteosarcoma of osteoblasts is a clinically common form at present, which generally occurs in the metaphysis of long-tubular bone, but seldom in the axial skeleton $(2,3)$. The malignant grade of osteosarcoma is high, the micro-lesion metastasis may be possible in the diagnosis and lung tissue is a common metastatic site (4). Before the application of chemotherapy drugs, amputation is generally used in the treatment of osteosarcoma. With the rise and development of chemotherapy drugs, cisplatin (CDDP), methotrexate and doxorubicin, have significant treatment effects on osteosarcoma, effectively improving the treatment level and cure rate of osteosarcoma $(5,6)$. The application of chemotherapy drugs is promising in the treatment of osteosarcoma, but the cure rate of osteosarcoma is generally about $60 \%$ due to chemotherapy drug resistance (7). Chemotherapy drug resistance is generally divided into endogenous drug resistance and acquired drug resistance, and there are various drug resistance-related molecular mechanisms, mainly including the increase of drug efflux, and changes in targets of drug metabolism and mutant drug therapy (8). Zhai et al (9) found that PAK5 and Ezrin genes are closely related to the occurrence and development of osteosarcoma, and patients with high expression of PAK5 and Ezrin genes are often prone to recurrence and metastasis with a poor chemotherapy effect. In the present study, the correlation between PAK5 and Ezrin genes and chemotherapy resistance of osteosarcoma patients was studied by in vivo and in vitro experimental systems, so as to clarify the related mechanism of osteosarcoma chemotherapy resistance from the molecular level and provide new ideas for the clinical prevention of osteosarcoma resistance. 


\section{Materials and methods}

Instruments and materials. Human osteosarcoma cell line SOSP-9607 (Shanghai Cell Bank, Chinese Academy of Sciences, Shanghai, China), methyl thiazolyltetrazolium (MTT) (Sigma; Merck KGaA, Darmstadt, Germany USA), dimethylsulfoxide (DMSO; Sigma; Merck KGaA), CDDP (Shanghai Aladdin Biochemical Technology Co., Ltd., Shanghai, China), TRIzol reagent (Invitrogen; Thermo Fisher Scientific, Inc., Waltham, MA, USA), reverse transcription kit (Invitrogen, Thermo Fisher Scientific, Inc.), ELISA kit (R\&D Systems, Inc., Minneapolis, MN, USA), ECI luminescent solution (Invitrogen, Thermo Fisher Scientific, Inc.), rabbit anti-human PAK5 polyclonal primary antibody (dilution, 1:1,000; cat. no. 3241), goat anti-rabbit polyclonal secondary antibody (dilution, 1:500; cat. no. 7074) (both from Cell Signaling Technology, Inc., Boston, MA, USA), rabbit anti-Ezrin and glyceraldehyde 3-phosphate dehydrogenase (GAPDH; Cell Signaling Technology, Inc., Danvers, MA, USA), inversed fluorescent microscope (Thermo Electron LED $\mathrm{GmbH}$, Langenselbold, Germany), cell culture flask (Corning Incorporated, Corning, NY, USA), pipettor (Eppendorf, Hamburg, Germany), Transwell chamber (Eppendorf), PCR instrument (ABI, Los Angeles, CA, USA), UV imaging system (Biometra $\mathrm{GmbH}$, Göttingen, Germany), electronic balance (BP121S; Sartorius AG, Göttingen, Germany) were obtained; other related equipment and reagents are described in the relevant parts. The study was approved by the Ethics Committee of Dezhou People's Hospital and informed consents were signed by the patients and/or guardians.

Construction of osteosarcoma drug-resistant cell lines. After human osteosarcoma cell line SOSP-9607 was resurrected, they were placed and incubated in an incubator. In vitro drugresistance cell lines of osteosarcoma were constructed through the induction of stepwise increasing concentration: The cells in the exponential growth phase were inoculated into the culture flask, and CDDP was added to induce concentrations of 0.1, $0.2,0.5,1$ and $2 \mu \mathrm{g} / \mathrm{ml}$; the induction was repeated for 5 times for each concentration; after cell culture for $48 \mathrm{~h}$, the medium was discarded and the fresh medium was added. The induction was repeated. After 12 months, the stable drug-resistance cell lines of osteosarcoma were constructed in the concentration of $2 \mu \mathrm{g} / \mathrm{ml}$, and frozen in liquid nitrogen for reserve.

Detection of cell growth cycle and drug sensitive test. Detection of cell growth cycle: The drug-resistance cell lines of osteosarcoma in the exponential growth phase were prepared into the suspension and inoculated into the culture dish in a concentration of $1 \times 10^{5}$ cells $/ 5 \mathrm{ml}$. The cells were collected every $24 \mathrm{~h}$ for a total of 7 days. The growth curve was drawn and the doubling time was detected. SOSP-9607 cell lines were used as the control group. Drug sensitivity test: The suspension of drug-resistant cell lines of osteosarcoma in the exponential growth phase was collected and inoculated

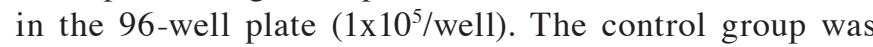
set-up according to the experimental requirements. CDDP in 8 different concentrations was added with 10-time increase for each concentration gradient. After cells were cultured for $72 \mathrm{~h}$, $5 \%$ MTT was added for another $4 \mathrm{~h}$; the culture medium was discarded and $150 \mu 1$ DMSO was added. The absorbance value at $492 \mathrm{~nm}$ was detected using the multi-functional microplate reader, $\mathrm{IC}_{50}$ value of each drug was calculated, and SOSP-9607 cell lines were used as the control group to determine whether the drug-resistance cell line was constructed successfully.

Detection of cell invasion capacity. The invasion capacity of cells was determined by Transwell assay: After drugresistant cell line of osteosarcoma SOSP-9607/DDP in good growing status was taken and starved for $2 \mathrm{~h}$, the cell number was adjusted into $5 \times 10^{5} / \mathrm{ml}$ and added into the Transwell chamber; and SOSP-9607 cell lines were used as the blank control group; after staining and fixation, the number of cells passing through the chamber was calculated under a microscope.

Detection of PAK5 and Ezrin gene expression levels via semi-quantitative PCR ( $q P C R)$. The total RNA was extracted from the drug-resistant cell lines of osteosarcoma SOSP-9607/CDDP and SOSP-9607 accurately according to the instructions of TRIzol kit. The RNA integrity was confirmed by agarose gel electrophoresis. The results of electrophoresis showed that $28 \mathrm{~S}, 18 \mathrm{~S}$ and $5 \mathrm{~S}$ bands were clear, and the brightness of $28 \mathrm{~S}$ band was about twice that of $18 \mathrm{~S}$ band, indicating that RNA is intact and can be used for subsequent experiments. cDNA was obtained via the reverse transcription using reverse transcription kit. The expression levels of PAK5 and Ezrin in tumor tissues and para-tumor tissues were detected by semi-qPCR with GAPDH as the internal reference. Reaction conditions: $95^{\circ} \mathrm{C}$ for $30 \mathrm{sec}, 64^{\circ} \mathrm{C}$ for $25 \mathrm{sec}$, $72^{\circ} \mathrm{C}$ for $30 \mathrm{sec}$, a total of 35 cycles. Primers were synthesized by Tiangen Biotech Co., Ltd. (Beijing, China). The sequences are shown in Table I. After the reaction, agarose gel electrophoresis was performed, followed by observation using UV imaging system.

Detection of PAK5 and Ezrin expression levels via western blot analysis. The proteins of drug-resistant cell lines of osteosarcoma SOSP-9607/CDDP and SOSP-9607 were extracted, and the supernatants were transferred to obtain the total protein after centrifugation. The protein samples were prepared into the sample loading system in the same concentration via protein quantification. Sodium dodecyl sulphate-polyacrylamide gel electrophoresis was used for sample loading. After membrane transfer, sealing and washing, the target bands were cut-off. The PAK5 and Ezrin primary antibodies were incubated overnight at $4^{\circ} \mathrm{C}$. The secondary antibody was incubated at room temperature for $2 \mathrm{~h}$ after washing with TBST (Tris-buffered saline Tween) three times. After washing with TBST three times, the target protein band was obtained via development exposure, followed by scanning and result analysis with GAPDH as the internal reference, so as to detect the expression quantities of PAK5 and Ezrin in osteoclastoma.

Detection of PAK5 and Ezrin expression in osteosarcoma tissues via immunohistochemistry. The paraffin sections of tumor tissue and para-tumor tissue samples of osteosarcoma patients were soaked with absolute ethyl alcohol, 95\% ethanol, $75 \%$ ethanol and distilled water for $10 \mathrm{~min}$, respectively, and washed twice with phosphate-buffered saline (PBS) 
Table I. PCR primer sequence of PAK5, Ezrin and GAPDH mRNA.

\begin{tabular}{ll}
\hline Gene & \multicolumn{1}{c}{ Sequence } \\
\hline PAK5 & F: 5'-ATCCACCTTGACGATGCTTTAC-3' \\
& R: 5'-TTCAGATGTTCTAAGCCTACGG-3' \\
\multirow{2}{*}{ Ezrin } & F: 5'-TGGCCCTCGTAGCCTTGAGGAC-3' \\
& R: 5'-CCAGTGCTGCAGGGTCCGAGGT-3' \\
GAPDH & F: 5'-GATGATTGGCATGGCTTT-3' \\
& R: 5'-CACCTTCCGTTCCAGTTT-3' \\
\hline
\end{tabular}

F, forward; R, reverse.

Table II. Sensitivity of SOSP-9607 and SOSP-9607/CDDP to cisplatin.

\begin{tabular}{lccc}
\hline & \multicolumn{2}{c}{$\mathrm{IC}_{50}($ mean $\pm \mathrm{SD}, \mu \mathrm{g} / \mathrm{ml})$} & \\
\cline { 2 - 3 } Agent & SOSP-9607 & SOSP-9607/CDDP & P-value \\
\hline CDDP & $3.28 \pm 0.42$ & $18.27 \pm 0.39$ & 0.0087 \\
\hline
\end{tabular}

CDDP, cisplatin; SD, standard deviation.

(5 min each time). After being soaked with $3 \% \mathrm{H}_{2} \mathrm{O}_{2}$ at room temperature for $10 \mathrm{~min}$, the samples were washed with PBS for $5 \mathrm{~min}$, and the confining liquid was dropped to seal the samples for $30 \mathrm{~min}$; after that, the redundant liquid was removed and PAK5 and Ezrin primary antibodies were added for incubation at $-4^{\circ} \mathrm{C}$ overnight, followed by washing with PBS for three times (5 min each time). Then the secondary antibody was added for incubation at room temperature for $1 \mathrm{~h}$, followed by washing with PBS three times. Then horseradish peroxidase was added for labeling for $30 \mathrm{~min}$, followed by development via DBA solution for $5 \mathrm{~min}$. At $30 \mathrm{~min}$ after the reaction was terminated using distilled water, hematoxylin was added for re-staining for 6-9 sec, followed by dehydration, transparency, mounting and microscopic examination.

Statistical analysis. The data are presented as mean \pm standard deviation and analyzed by SPSS 19.0 (SPSS, Inc., Chicago, IL, USA) software. t-test was used for measurement data, while Chi-square test was used for enumeration data. Pearson's analysis was used for correlation analysis. One-way analysis of variance (ANOVA) was used for other data. Bonferronic method was used for pairwise comparison under homogeneity of variance, while Welch method was used under heterogeneity of variance. Dunnett's T3 method was used for multiple comparisons.

\section{Results}

Cell growth cycle and doubling time. The drug-resistance cell line of osteosarcoma SOSP-9607/CDDP was constructed, and cell line SOSP-9607 was used as the control group. The growth cycle and doubling time of cells in each group were detected

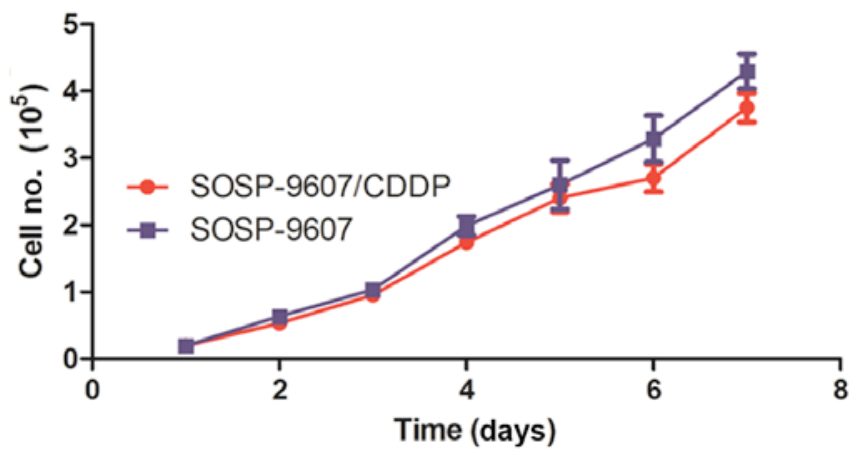

Figure 1. The growth curves of SOSP-9607/CDDP and SOSP-9607; the growth rate of SOSP-9607 was similar to that of SOSP-9607/DDP. The doubling time of SOSP-9607/CDDP was similar to that of SOSP-9607 $(\mathrm{P}>0.05)$.

by MTT assay. The results are showed in Fig. 1: The growth rate of SOSP-9607 was similar to that of SOSP-9607/DDP, with incubation time as the $\mathrm{x}$-axis and average cell count as the $y$-axis, and the difference was not statistically significant (P>0.05). The doubling time of SOSP-9607/CDDP was similar to that of SOSP-9607, and the difference was not statistically significant $(\mathrm{P}>0.05)$.

Sensitivity of SOSP-9607/CDDP and SOSP-9607 to drugs. CDDP in 8 different concentrations was added into the drug-resistance cell line of osteosarcoma SOSP-9607/CDDP. SOSP-9607 was used as the control group. The sensitivity of SOSP-9607/CDDP to the above drug was detected via MTT assay and $\mathrm{IC}_{50}$ value was calculated, and the results are shown in Table II. The results showed that $\mathrm{IC}_{50}$ values of SOSP-9607 and SOSP-9607/CDDP were 3.28 \pm 0.42 and $18.27 \pm 0.39$, and the difference was statistically significant $(\mathrm{P}<0.01)$.

Detection of cell migration capacity. The migration capacity of drug-resistance cell line of osteosarcoma SOSP-9607/CDDP was detected via Transwell assay. SOSP-9607 was used as the control group. The results are shown in Fig. 2: The migration capacity of SOSP-9607/CDDP was significantly increased compared with that of SOSP-9607, and the difference was statistically significant $(\mathrm{P}<0.01)$.

Detection of PAK5 and Ezrin expressions via semi-qPCR. The expression levels of PAK5 and Ezrin in SOSP-9607/CDDP and SOSP-9607 were detected by semi-qPCR. The results are shown in Fig. 3: The expression levels of PAK5 and Ezrin in SOSP9607/CDDP were significantly higher than those in SOSP-9607, and the differences were statistically significant $(\mathrm{P}<0.01)$.

Detection of PAK5 and Ezrin expression via western blot analysis. The expression levels of PAK5 and Ezrin in SOSP-9607/CDDP and SOSP-9607 were detected via western blot analysis. The results are shown in Fig. 4: The PAK5 and Ezrin protein expression levels in SOSP-9607/CDDP were significantly higher than those in SOSP-9607, and the differences were statistically significant $(\mathrm{P}<0.01)$.

Detection of PAK5 and Ezrin expression in osteosarcoma tissues via immunohistochemistry. The expression levels of 
A SOSP-9607/CDDP

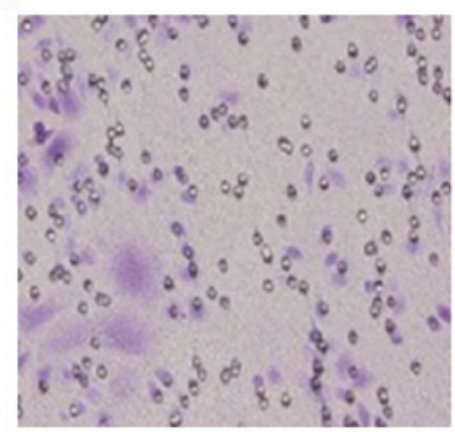

B

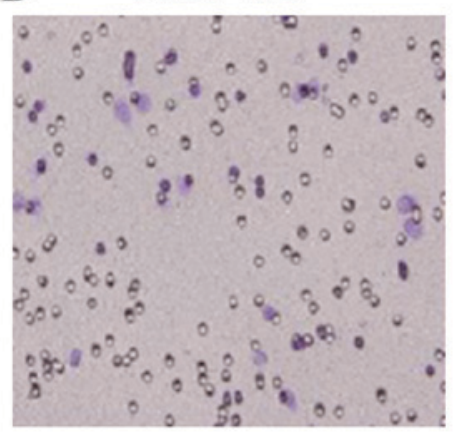

C

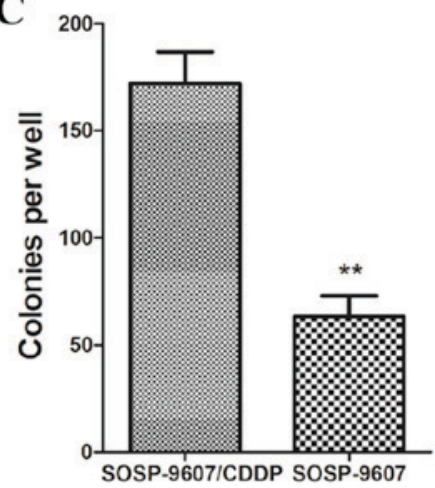

Figure 2. Detection of migration capacity of SOSP-9607/CDDP via Transwell assay. (A) Micrograph of SOSP-9607/CDDP. (B) Micrograph of SOSP-9607. (C) Statistical chart; the results showed that the migration capacity of SOSP-9607/CDDP was significantly increased compared with that of SOSP-9607 ( $\left.{ }^{* *} \mathrm{P}<0.01\right)$.

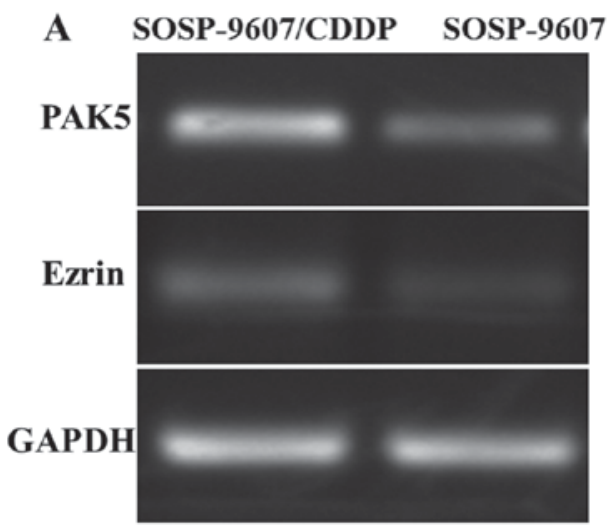

B

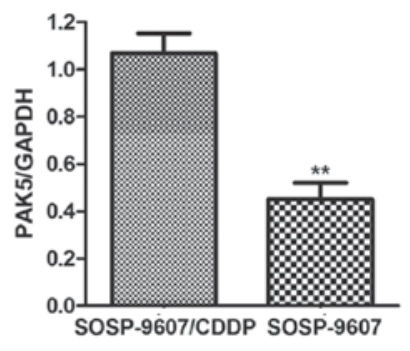

C

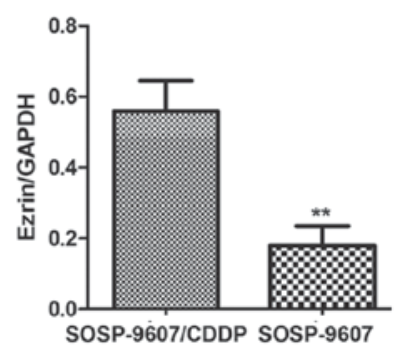

Figure 3. Detection of PAK5 and Ezrin expression via semi-quantitative PCR. (A) Agarose gel electrophoresis chart. (B and C) Statistical charts of relative expression quantities of PAK5 and Ezrin; the results showed that PAK5 and Ezrin expression levels in SOSP-9607/CDDP was significantly higher than those in SOSP-9607 (**P $<0.01)$.

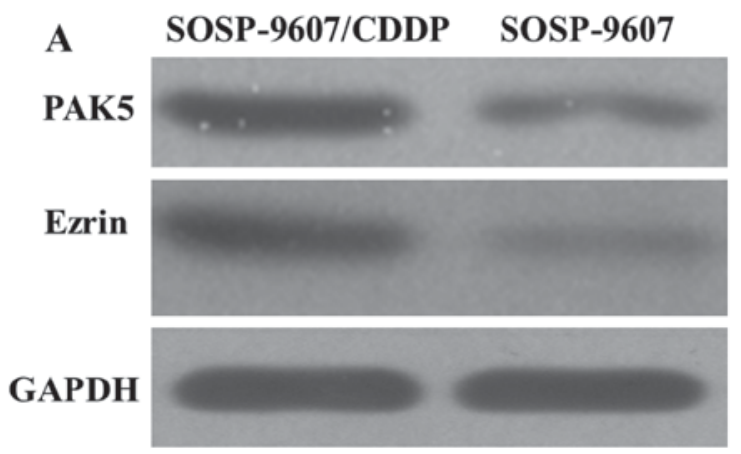

B

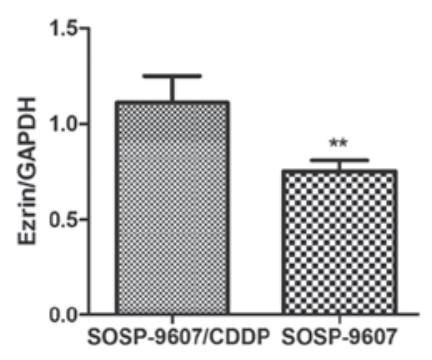

C

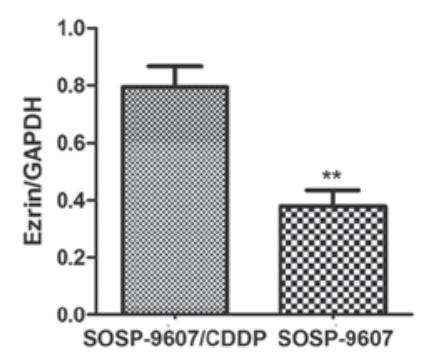

Figure 4. Detection of PAK5 and Ezrin protein expression levels via western blot analysis. (A) Bar graph of western blot analysis. (B and C) Statistical charts of PAK5 and Ezrin protein expression quantities. The results showed that PAK5 and Ezrin protein expression levels in SOSP-9607/CDDP were significantly higher than those in SOSP-9607 $\left({ }^{* *} \mathrm{P}<0.01\right)$.

PAK5 and Ezrin in osteosarcoma were detected via immunohistochemistry. The para-tumor tissue was used as the control group. Yellow staining in cytoplasm and surrounding mesenchyme was regarded as PAK5-positive expression, while brown staining in nucleus was regarded as Ezrin-positive expression. The immunohistochemical results of PAK5 in osteosarcoma and para-tumor tissue are shown in Fig. 5: The expression level of PAK5 in osteosarcoma was significantly higher than that in para-tumor tissue, and the difference was statistically significant $(\mathrm{P}<0.01)$. The immunohistochemical results of Ezrin in osteosarcoma and para-tumor tissue are shown in Fig. 6: The expression level of Ezrin in osteosarcoma was significantly higher than that in para-tumor tissue, and the difference was statistically significant $(\mathrm{P}<0.01)$.

Analysis of correlation between PAK5 and Ezrin expression levels in osteosarcoma patients. The CDDP resistance situation of 78 osteosarcoma patients was recorded, the 
A Osteosarcoma tissue

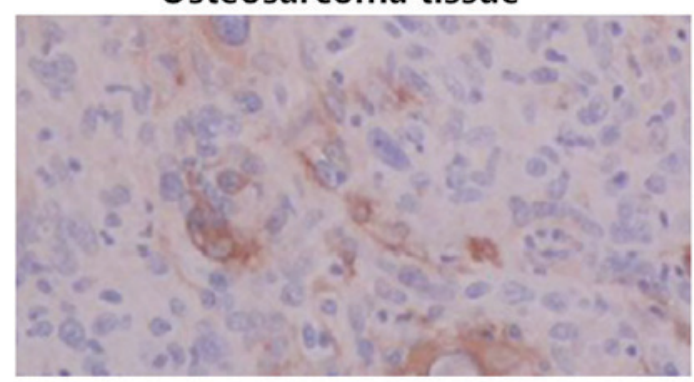

B

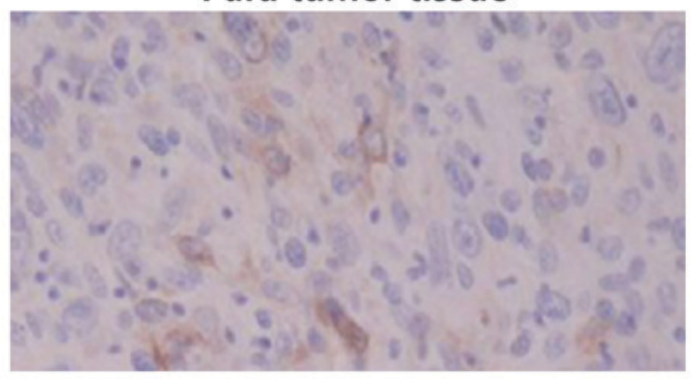

Figure 5. Detection of PAK5 expression via immunohistochemistry. (A) PAK5 expression level in osteosarcoma. (B) PAK5 expression level in para-tumor tissue; the results showed that PAK5 positive expression rate in osteosarcoma was significantly higher than that in para-tumor tissue.

A

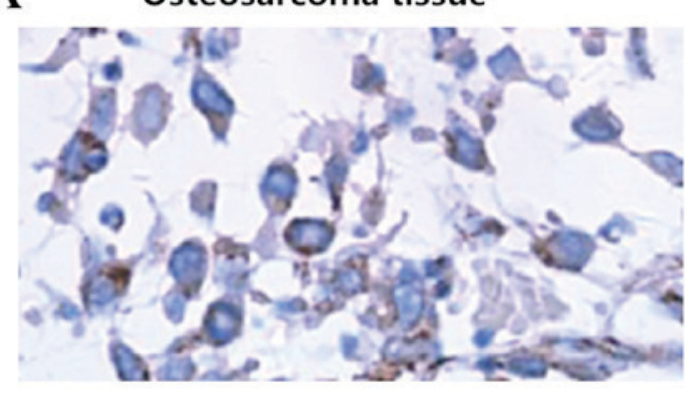

B

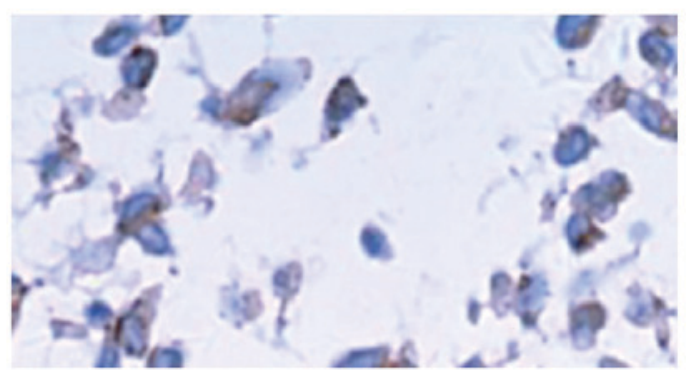

Figure 6. Detection of Ezrin expression via immunohistochemistry. (A) Ezrin expression level in osteosarcoma. (B) Ezrin expression level in para-tumor tissue; the results showed that Ezrin positive expression rate in osteosarcoma was significantly higher than that in para-tumor tissue.

Table III. Correlation between PAK5 and Ezrin expression in osteosarcoma tissues.

\begin{tabular}{lcccc}
\hline & \multicolumn{2}{c}{ PAK5 } & & \\
\cline { 2 - 3 } Ezrin & Positive & Negative & r & P-value \\
\hline Positive & 38 & 13 & 0.197 & 0.023 \\
Negative & 32 & 9 & & \\
\hline
\end{tabular}

positive rates of PAK and Ezrin were detected and correlation analysis was performed for the expressions of PAK and Ezrin. Pearson's correlation analysis showed that the expressions of PAK and Ezrin were positively correlated in drug resistance of osteosarcoma $(r=0.197, \mathrm{P}=0.023)$. The results are shown in Table III.

\section{Discussion}

Osteosarcoma is the most common malignant bone tumor in clinic at present, which often occurs in young people, and its main clinical manifestations are progressively aggravated bone pain and local swelling $(10,11)$. The 5-year survival rate of osteosarcoma patients is low, placing great economic and mental burdens on the society and family (12). The chemotherapy drug resistance in the treatment process of osteosarcoma is the problem puzzling clinicians currently. There is related literature reporting that the expression levels of some important genes and proteins not only affect the occur- rence and development of osteosarcoma, but also are closely related to the clinical treatment effect of osteosarcoma (13).

PAK is an evolutionarily highly conserved protein kinase, and it plays an important role in occurrence, invasion and metastasis of tumors (14). PAK5 has been seldom studied, and it is a newly-discovered member in PAK family, which is capable of activating the cell survival signaling pathway and protecting the cell survival (15). Previous studies have shown that PAK5 may play an important role in the development and progression of malignant tumors. Gu et al (16) detected the expression of PAK5 in tumor tissues of osteosarcoma patients via immunohistochemistry, and found that PAK5 was highly expressed in osteosarcoma patients, suggesting that PAK5 may be related to the pathogenesis of osteosarcoma. Ezrin protein is a member of the cytoskeletal cross-linked protein ERM, which mainly mediates the connective function of membrane and cytoskeleton with a series of functions for relevant cells (17). The excessive expression and activation of Ezrin can lead to abnormal intercellular signal transmission and promote tumor cell metastasis, thus playing a role in tumor invasion and metastasis $(18,19)$. Studies have shown that Ezrin protein is closely related to pulmonary metastasis of osteosarcoma patients, which can be used as an index of predicting the prognosis and survival (20). In the present study, the expression levels of PAK5 and Ezrin in tumor tissues and para-tumor tissues of patients with osteosarcoma resistance were analyzed by western blot analysis. The results showed that the expression levels of PAK5 and Ezrin in osteosarcoma tissues were significantly higher than those in para-tumor tissues, suggesting that the drug resistance of osteosarcoma 
patients may be closely related to the high expression of PAK5 and Ezrin. The CDDP-resistance osteosarcoma cell line was constructed and the expression of PAK5 and Ezrin was detected via semi-qPCR and western blot analysis from the gene and protein level. The results showed that the expression quantities of PAK5 and Ezrin in CDDP-resistance osteosarcoma cell line were significantly higher than those in osteosarcoma cell line, which was consistent with the results in osteosarcoma patients. The above results revealed in vivo and in vitro that PAK5 and Ezrin are closely related to the drug resistance of osteosarcoma patients. The correlation study on expression of PAK5 and Ezrin showed that the expression of PAK5 and Ezrin are positively correlated $(r=0.197, \mathrm{P}=0.023)$, suggesting that PAK5 and Ezrin may affect the drug resistance of osteosarcoma to chemotherapy drugs through synergistic effect.

In conclusion, PAK5 and Ezrin are closely related to the drug resistance of osteosarcoma, which provides clinical reference for clinically clarifying drug resistance mechanism of osteosarcoma. However, there were still some short comings in this study: Related mechanism of PAK5 and Ezrin involved in drug resistance of osteosarcoma was not studied deeply, the sample size in this experiment was small and the comparative study was not performed for healthy volunteer samples, so the results need to be further confirmed. However, the research values of PAK5 and Ezrin in drug resistance of osteosarcoma are beyond doubt, which can bring a new breakthrough for clinical chemotherapy of osteosarcoma.

\section{References}

1. Shimizu T, Kido A, Honoki K, Murata K, Fujii H, Higuchi B Ishihara T, Takeshita Y, Shima M, Yajima H, et al: A successful reconstruction using a frozen autograft and a pedicled latissimus dorsi flap after a S12345B shoulder girdle resection in a patient with osteosarcoma. J Reconstr Microsurg 28: 155-159, 2012.

2. Qiu Q, Jiang J, Lin L, Cheng S, Xin D, Jiang W, Shen J and Hu Z: Downregulation of RSK2 influences the biological activities of human osteosarcoma cells through inactivating AKT/mTOR signaling pathways. Int J Oncol 48: 2508-2520, 2016.

3. Hurley C, McCarville MB, Shulkin BL, Mao S, Wu J, Navid F, Daw NC, Pappo AS and Bishop MW: Comparison of (18) F-FDG-PET-CT and bone scintigraphy for evaluation of osseous metastases in newly diagnosed and recurrent osteosarcoma. Pediatr Blood Cancer 63: 1381-1386, 2016.

4. Waresijiang N, Sun J, Abuduaini R, Jiang T, Zhou W and Yuan H: The downregulation of miR 125a 5p functions as a tumor suppressor by directly targeting MMP 11 in osteosarcoma. Mol Med Rep 13: 4859-4864, 2016.

5. Zeng W, Liu Q, Chen Z, Wu X, Zhong Y and Wu J: Silencing of hERG1 gene inhibits proliferation and invasion, and induces apoptosis in human osteosarcoma cells by targeting the NF- $\kappa \mathrm{B}$ Pathway. J Cancer 7: 746-757, 2016.
6. Sun Y, Xia P, Zhang H, Liu B and Shi Y: P53 is required for Doxorubicin-induced apoptosis via the TGF-beta signaling pathway in osteosarcoma-derived cells. Am J Cancer Res 6: 114-125, 2015.

7. Zheng K, Yu X, Chang Z, Xu S and Xu M: Effect of pathological fracture on limb salvage surgery with preservation of the epiphysis in children with osteosarcoma of the distal femur: Two case reports. Mol Clin Oncol 4: 523-526, 2016.

8. Zhao J, Chen F, Zhou Q, Pan W, Wang X, Xu J, Ni L and Yang H: Aberrant expression of microRNA-99a and its target gene mTOR associated with malignant progression and poor prognosis in patients with osteosarcoma. Onco Targets Ther 9: 1589-1597, 2016

9. Zhai J, Qu S, Li X, Zhong J, Chen X, Qu Z and Wu D: miR-129 suppresses tumor cell growth and invasion by targeting PAK 5 in hepatocellular carcinoma. Biochem Biophys Res Commun 464: 161-167, 2015.

10. Bao ZQ, Zhang CC, Xiao YZ, Zhou JS, Tao YS and Chai DM: Over-expression of Sox 4 and $\beta$-catenin is associated with a less favorable prognosis of osteosarcoma. J Huazhong Univ Sci Technolog Med Sci 36: 193-199, 2016.

11. Maximov VV and Aqeilan RI: Genetic factors conferring metastasis in osteosarcoma. Future Oncol 12: 1623-1644, 2016.

12. Zhou Q, Shen L, Liu C, Liu C, Chen H and Liu J: The effects of estradiol and glucocorticoid on human osteosarcoma cells: Similarities and differences. Anticancer Res 36: 1683-1691, 2016.

13. Dai Y, Jiang J, Wang Y, Jin Z and Hu S: The correlation and clinical implication of VEGF-C expression in microvascular density and lymph node metastasis of gastric carcinoma. Am J Transl Res 8: 5741-5747, 2016.

14. Huang JF, Du WX and Chen JJ: Elevated expression of matrix metalloproteinase-3 in human osteosarcoma and its association with tumor metastasis. J BUON 21: 1279-1286, 2016.

15. Li Y, Ke Q, Shao Y, Zhu G, Li Y, Geng N, Jin F and Li F: GATA1 induces epithelial-mesenchymal transition in breast cancer cells through PAK5 oncogenic signaling. Oncotarget 6: 4345-4356, 2015.

16. Gu X, Wang C, Wang X, Ma G, Li Y, Cui L, Chen Y, Zhao B and $\mathrm{Li} \mathrm{K}$ : Efficient inhibition of human glioma development by RNA interference-mediated silencing of PAK5. Int J Biol Sci 11: 230-237, 2015.

17. Pan D, Wang S, Ye H, Xu S and Ye G: Ezrin expression in the primary hepatocellular carcinoma patients and associated with clinical, pathological characteristics. J Cancer Res Ther 12: 291-294, 2016.

18. Du P, Xu B, Zhang D, Shao Y, Zheng X, Li X, Xiong Y, Wu C and Jiang J: Hierarchical investigating the predictive value of p53, COX2, EGFR, nm23 in the post-operative patients with colorectal carcinoma. Oncotarget 8: 954-966, 2017.

19. Rox K, Rohde M, Chhatwal GS and Müller R: Disorazoles block group A streptococcal invasion into epithelial cells via interference with the host factor Ezrin. Cell Chem Biol 24: 159-170, 2017.

20. Hago AM, Gamallat Y, Mahmoud SA, Huang Y, Zhang J, Mahmoud YK, Wang J, Wei Y, Wang L, Zhou S, et al: Ezrin expression is altered in mice lymphatic metastatic hepatocellular carcinoma and subcellular fractions upon Annexin 7 modulation in-vitro. Biomed Pharmacother 85: 209-217, 2017.

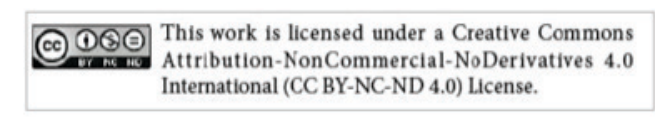

